\section{Cigarette Smoking Initiation among Substance Abuse Treatment Population: How applicable is the Gateway Hypothesis?}

Keywords: Smoking initiation; Smoking progression; Substance abuse; Treatment; Gateway hypothesis

\begin{abstract}
Background: Cigarette smoking has been considered a gateway to subsequent, and often, co-occuming usage of other lic it or illic it substances in the general population. However, significant deviations from this typical pattem of progression have been observed among substance abusers. Moreover, progression pattems may differ by demographicsand substance abuse-related risk factors.
\end{abstract}

Objectives: This research examines the pattems of cigarette smoking initiation among substance abusers; and explores the correlates of cigarette smoking with demographic and other substance abuse-related factors with different pattems of cigarette smoking initiation in the substance abuse treatment population.

Methods: The sample was comprised of 1,179 substance abuse clients, who received publicly-funded treatment in Tennessee. Data were collected from the clients at intake and six-months post intake.

Results: A significant proportion of clients reported cigarette smoking (82\%). Only 34\% clients reported typical progression pattems (i.e. cigarette as a gateway substance). Others reported deviations in smoking progression pattems with $48 \%$ atypic al progression - ciga rette smoking initiated post substance use, and $18 \%$ reporting as neversmokers. African Americans and college-educated partic ipants were more likely to be atypical progressors or never-smokers. Manijuana users were more likely to be atypical progressors. Also, those who initiated lic it or illic it drug use at an earlier age were more likely to be either atypic al progressors or never-smokers.

Conc lusions: While the GatewayHypothesis $(\mathrm{GH})$ still hold sfora bout a third of this population, these results suggest that it is insufficient in explaining cigarette smoking initiation among problematic substance users. In addition, the high prevalence of cigarette smoking in this population suggests the need for tobacco cessation efforts, a longside substance abuse treatment. In addition, the development of tailored preventative programs for those at greatest risk is needed to ward off gateway substances preceding problematic illic it drug abuse.

\section{Introduction}

The Gateway Hypothesis (GH) has dominated the debate on the progression of substance abuse in the literature [1]. This framework delineates the progression pattern from the use of one or more licit substances to the subsequent use of other licit or illicit substances [2]. Originally, and still at times, tobacco and alcohol are held as the licit gateway substances that precede marijuana, which in turn, lead to the subsequent use of other illicit drugs [2,3]. Some studies have provided

\section{Journal of}

Addiction \& Prevention

Paige Pirkey ${ }^{1}$, Satish Kedia ${ }^{1 *}$, Larita Webb ${ }^{1}$, George Relyea ${ }^{1}$, Mohammad Masudul Alam ${ }^{1}$, and Fawaz Mzayek $^{2}$

${ }^{1}$ Division of Social \& Behavioral Sciences, School of Public Health, University of Memphis, USA

${ }^{2}$ Division of Epidemiology, Biostatistics, \& Environmental Health, School of Public Health, University of Memphis, USA

\section{*Address for Correspondence}

Satish Kedia, PhD, Professor, Division of Social \& Behavioral Sciences, School of Public Health, University of Memphis, 3825 Desoto Avenue, Memphis, Tennessee, 38152, USA, Tel: 901.678.1433; Fax: 901.678.1715; E-mail: skkedia@memphis.edu

Submission: 01 December, 2015

Accepted: 24 December, 2015

Published: 28 December, 2015

Copyright: $\odot 2015$ Pirkey $P$, et al. This is an open access article distributed under the Creative Commons Attribution License, which permits unrestricted use, distribution, and reproduction in any medium, provided the original work is properly cited.

Reviewed \& Approved by: Dr. Thomas Heffernan, Department of Psychology and Faculty of Life and Health Sciences, Northumbria University, UK

evidence in support of GH confirming that these gateway substances predict subsequent, illicit drug use [3-7]. However, deviations in substance use progression patterns have been reported and reflect confounding variables such as gender, ethnicity, age, education level, employment status, and mental disorders, be associated with varying substance abuse progression patterns [8-13]. Mackesy-Amiti et al. reported deviations or atypical progression patterns among persons from disadvantaged backgrounds i.e., those with less than a high school education, living in a shelter, unemployed, and whose drug use began at a younger age [11]. These deviations in substance abuse progression patterns by individual and environmental factors point to obvious limitations of the GH framework. The foremost limitations are grounded in the central features of the GH: sequence, association, and causation [14]. The sequence paradigm asserts that the onset of a gateway substance antedates the initiation of other, harder drugs, and this existent association in substance use increases the risk of not only using these harder drugs, but also of developing problematic substance use. Indeed, sequence and association indicate that substance use initiation and subsequent developmental stages are progressive and hierarchical, which inherently implies a causality constituent [2]. Contrary to GH assertions, deviations have been reported. For instance, the lack of, or postponement in, the initiation of a gateway substance, such as tobacco, has not been found to decrease the likelihood of subsequent illicit drug use [15].

Tobacco is still an important addictive substance, regardless of whether or not it is a gateway drug. Two systematic reviews of 94 published papers revealed that the majority of substance abusers being treated for drug abuse also reported cigarette smoking $[16,17]$. As reported for any given year in both studies (either between 1987 and 2009 or between 1987 and 2013), the prevalence of cigarette 
smoking among the substance abuse treatment population exceeded $80 \%$, which greatly surpassed the prevalence observed in the general population [17,18]. Moreover, smoking cessation efforts, alongside substance abuse treatment programs, have become an emergent topic of interest [19]. The increased concern and desire to quit smoking has been documented among substance abuse treatment clients [20,21] Therefore, studying tobacco among substance abusers is warranted and underscored by the increased likelihood of people dying from smoking-related complications compared to other causes [22-24].

Clearly, more research is needed to identify patterns of cigarette smoking initiation among substance abusers. Understanding the correlates of cigarette smoking patterns with demographic characteristics and other risk factors may reveal opportunities for targeted smoking cessation interventions along with substance abuse treatment. For instance, using a gateway substance before initiating illicit substance use is less prevalent among Asian (12.5\%), Hispanic (21.9\%), and African Americans (23.3\%) substance abusers compared to non-Hispanic white substance abusers (29.5\%), which may create a potential opportunity for targeted interventions for certain ethnic groups [25]. This study examined (1) the patterns of cigarette smoking initiation among substance abuse treatment clients, and (2) the correlates of cigarette smoking with demographic and other substance abuse-related factors with different patterns of cigarette smoking initiation in this population.

\section{Research Methodology}

\section{Design and study participants}

The study employed a cross-sectional survey design among substance abuse clients six months after their admissions to a publicly-funded treatment facility in Tennessee. This study was part of a Substance Abuse and Mental Health Services Administrator (SAMHSA) funded outcomes research which was administered through the Tennessee Department of Mental Health. The participants in the study were 13 years of age or older, resided in Tennessee, and received substance abuse treatment between July and December in 2004 in any of the 110 treatment facilities located throughout the state. Also, since the publicly-funded program primarily supported indigent clients, all participants were below the poverty line, which in 2004 was $\$ 18,850$ for a family of four, as specified by the Poverty Guidelines of the United States Department of Health and Human Services (HHS). Written informed consent to participate in the follow-up interview was collected from adult clients or legal guardians/parents of children younger than 18 years. The University of Memphis Institutional Review Board approved the study and consent forms.

To examine the patterns of cigarette smoking initiation among the substance abuse clients, following Mackesy-Amiti et al. study participants were divided into three categories: Typical progressors those who used cigarettes/alcohol, followed by illicit drugs; atypical progressors - those who used illicit drugs prior to cigarette/alcohol followed by substance use, and never-smokers [11].

\section{Procedures}

While the intake data were collected at the time of admission by the facility staff, the six-month follow-up data were collected via telephone by trained interviewers at the University of Memphis
Institute for Substance Abuse Research and Evaluation (I-SARE) between January and June 2005. The interviewers attempted to contact those who provided informed consent to participate in the study. However, clients who either refused to participate, provided no telephone number, listed wrong telephone numbers, or were institutionalized (e.g., in prison, state custody, a hospital, shelter, or group home), still in treatment, or deceased at the time of six-month follow-up were excluded from the study. Interviewers made no fewer than seven attempts (three during the day, three during the evening, and one over the weekend) to contact clients for optimal participation.

\section{Measures}

Demographic data (i.e., gender, ethnicity, age, education level, employment status, income, marital status, living arrangement, dependents, and residential location) were compiled from the follow-up data. Substance abuse data were collected both at intake and at six-month follow-up using a questionnaire based on the Addiction Severity Index (ASI) [26,27], which included questions about age of onset of substances abused-namely, alcohol, cocaine (powder and "crack" cocaine), marijuana, opiates/narcotics (e.g., heroin, morphine, methadone, and Demerol), sedatives/hypnotics (e.g., benzodiazepines and barbiturates), stimulants/amphetamines (e.g., methamphetamine and Dexedrine), hallucinogens (e.g., LSD), and other (e.g., "club drugs," such as Ecstasy, inhalants, and PCP). Whether the client received treatment for only substance abuse or for substance abuse along with a concurrent mental health condition was extracted from intake data. Questions related to family history of substance abuse were also collected at intake and six-month followup. Smoking history and behavior information were collected using a 14-item questionnaire developed by smoking cessation experts at the University of Memphis [28].

\section{Data analysis}

Descriptive statistics were reported as percentages or means with standard deviations. Comparisons between the three categories were made using ANOVA or chi-square tests, as appropriate. Unadjusted odds ratios (ORs) were calculated using logistic regression analysis; adjusted ORs were determined via multivariate logistic analyses. Separate models were used to compare atypical vs. typical progressors and never-smokers vs. typical progressors. The model included demographics (gender, ethnicity, educational level, and residential location), substance use variables (age of onset, number and type of substances used, whether treated for substance abuse and/or cooccurring mental health issues), and family history of substance abuse as independent variables. To assess the role of substance abuse behavior, a 3-level dummy-coded summary variable was created for number and types of substances used at time of admission (alcohol use only, a single illicit drug only, and multiple substances). The dependent variable was self-reported history of cigarette smoking initiation: typical, atypical, and never-smokers.

\section{Results}

\section{Socio-demographics and patterns of onset of cigarette smoking}

Of 1,179 participants in this study, about $68 \%$ were male and $76 \%$ were non-Hispanic white. The mean age of participants entering treatment was approximately 34 years old. Twenty percent 
Citation: Pirkey P, Kedia S, Webb L, Relyea G, Alam MM, et al. Cigarette Smoking Initiation among Substance Abuse Treatment Population: How applicable is the Gateway Hypothesis? J Addiction Prevention. 2015;3(2): 7.

ISSN: $2330-2178$

of participants had a college degree, while $69 \%$ had only a high school education or less. Nearly half (48\%) of participants reported earning less than $\$ 2,000$ the prior year. More than half of participants reported living in a rural area (56\%). Also, most reported having no dependents (61\%) and almost half being single and never married (48\%). Thirty four percent of participants reported being typical progressors, $48 \%$ atypical progressors, and $18 \%$ were never-smokers (see Table 1). As for age of onset of cigarette smoking and substance

Table 1: Socio-demographics and other factors by patterns of cigarette smoking $(n=1,179)$.

\begin{tabular}{|c|c|c|c|c|}
\hline & $\begin{array}{l}\text { Total Sample } \\
\mathrm{N}=1,179\end{array}$ & $\begin{array}{l}\text { Typical Progressors } \\
\qquad n=402\end{array}$ & $\begin{array}{l}\text { Atypical Progressors } \\
\qquad n=564\end{array}$ & $\begin{array}{l}\text { Never Smokers } \\
n=213\end{array}$ \\
\hline & $(100 \%)$ & $(34.1 \%)$ & $(47.8 \%)$ & $(18.1 \%)$ \\
\hline Client Characteristics & & n (\%) & n (\%) & n (\%) \\
\hline \multicolumn{5}{|l|}{ Socio-demographics } \\
\hline \multicolumn{5}{|l|}{ Gender $^{\mathrm{b}}$} \\
\hline Female & $384(32.6)$ & $153(38.1)$ & $174(30.8)$ & $57(26.8)$ \\
\hline Male & $795(67.4)$ & $249(61.9)$ & $390(69.1)$ & $156(73.2)$ \\
\hline \multicolumn{5}{|l|}{ Ethnicityc } \\
\hline African American & $278(23.6)$ & $76(18.9)$ & $130(23.1)$ & $72(33.8)$ \\
\hline Caucasian & $901(76.4)$ & $326(81.1)$ & $434(76.9)$ & $141(66.2)$ \\
\hline Age $^{e}$ (years) & 33.97 (10.5 SD) & $34.22(10.2$ SD) & 33.73 (10.3 SD) & $34.03(11.7 \mathrm{SD})$ \\
\hline \multicolumn{5}{|l|}{ Residential locationa } \\
\hline Urban & $514(43.6)$ & $165(41.0)$ & $233(41.3)$ & $116(54.5)$ \\
\hline Rural & $665(56.4)$ & $237(59.0)$ & $331(58.7)$ & $97(45.5)$ \\
\hline \multicolumn{5}{|l|}{ Education ${ }^{\mathrm{a}, 1}$} \\
\hline Middle/High School & $813(69.0)$ & $291(72.4)$ & $395(70.0)$ & $127(59.6)$ \\
\hline College & $238(20.2)$ & $68(16.9)$ & $115(20.4)$ & $55(25.8)$ \\
\hline \multicolumn{5}{|l|}{ Current employment status ${ }^{\mathrm{e}, 2}$} \\
\hline Full & $230(19.5)$ & $76(18.9)$ & $102(18.1)$ & $52(24.4)$ \\
\hline Other & $930(78.9)$ & $317(78.9)$ & $454(80.5)$ & $159(74.6)$ \\
\hline \multicolumn{5}{|l|}{ Past year income ${ }^{e}$} \\
\hline$<\$ 2,000$ & $570(48.4)$ & $209(52.0)$ & $265(47)$ & $96(45.1)$ \\
\hline$\$ 2,000-\$ 8,500$ & $243(20.6)$ & $81(20.2)$ & $124(22)$ & $38(17.8)$ \\
\hline$>\$ 25,000$ & $69(5.9)$ & $16(4.0)$ & $32(5.7)$ & $21(9.9)$ \\
\hline \multicolumn{5}{|l|}{ Marital status ${ }^{d}$} \\
\hline Married & $155(13.2)$ & $45(11.2)$ & $77(13.7)$ & $33(15.5)$ \\
\hline Single never been married & $562(47.7)$ & $182(45.3)$ & $268(47.5)$ & $112(52.6)$ \\
\hline Divorced/Separated & $462(39.2)$ & $175(43.5)$ & $219(38.8)$ & $68(31.9)$ \\
\hline \multicolumn{5}{|l|}{ Current living arrangement ${ }^{\mathrm{c}, 3}$} \\
\hline Living Alone & $276(23.4)$ & $96(23.9)$ & $146(25.9)$ & $34(16)$ \\
\hline Living with Others & $881(74.7)$ & $299(74.4)$ & $405(71.8)$ & $177(83.1)$ \\
\hline \multicolumn{5}{|l|}{ Total no. of dependents ${ }^{\mathrm{d}, 4}$} \\
\hline None & $723(61.3)$ & $232(57.7)$ & $345(61.2)$ & $146(68.5)$ \\
\hline $1-2$ & $336(28.5)$ & $127(31.6)$ & $165(29.3)$ & $44(20.7)$ \\
\hline $3-5$ & $119(10.1)$ & $43(10.7)$ & $54(9.6)$ & $22(10.3)$ \\
\hline \multicolumn{5}{|l|}{ Smoking History } \\
\hline \multicolumn{5}{|c|}{ Age of onset of cigarette smoking } \\
\hline $6-12$ & $200(20.7)$ & $138(34.3)$ & $62(11.0)$ & --- \\
\hline $13-18$ & $614(63.6)$ & $244(60.7)$ & $370(65.6)$ & --- \\
\hline
\end{tabular}


Citation: Pirkey P, Kedia S, Webb L, Relyea G, Alam MM, et al. Cigarette Smoking Initiation among Substance Abuse Treatment Population: How applicable is the Gateway Hypothesis? J Addiction Prevention. 2015;3(2): 7.

ISSN: $2330-2178$

\begin{tabular}{|c|c|c|c|c|}
\hline $19+$ & $152(15.7)$ & $20(5.0)$ & $132(23.4)$ & --- \\
\hline \multicolumn{5}{|l|}{ Substance abuse-related factors } \\
\hline $6-12$ & $205(17.4)$ & $30(7.5)$ & $148(26.3)$ & $27(12.7)$ \\
\hline $13-18$ & $616(52.3)$ & $166(41.3)$ & $336(59.5)$ & $114(53.5)$ \\
\hline \multicolumn{5}{|l|}{ Number of substances used at intake ${ }^{a}$} \\
\hline Alcohol only & $259(22.0)$ & $67(16.7)$ & $136(24.1)$ & $56(26.3)$ \\
\hline Single illicit drug only & $262(22.2)$ & $141(35.1)$ & $60(10.6)$ & $61(28.6)$ \\
\hline Multiple substances used & $658(55.8)$ & $194(48.3)$ & $368(65.3)$ & $96(45.1)$ \\
\hline \multicolumn{5}{|l|}{ Drug use reported at intake ${ }^{f}$} \\
\hline Cocaine $^{a}$ & $491(41.7)$ & $181(45.0)$ & $236(41.8)$ & $74(34.7)$ \\
\hline Marijuana $^{c}$ & 447 (37.9) & $101(25.1)$ & $271(48.1)$ & $75(35.2)$ \\
\hline Opiates/ narcotics ${ }^{c}$ & $243(20.6)$ & $109(27.1)$ & $102(18.1)$ & $32(15.0)$ \\
\hline Sedatives/hypnotics ${ }^{e}$ & $129(10.9)$ & $53(13.2)$ & $58(10.3)$ & $18(8.5)$ \\
\hline Stimulants/ amphetamines ${ }^{b}$ & $119(10.1)$ & $54(13.4)$ & $54(9.6)$ & $11(5.2)$ \\
\hline Hallucinogens/other drugs ${ }^{a}$ & $45(3.8)$ & $7(1.7)$ & $27(4.8)$ & $11(5.2)$ \\
\hline \multicolumn{5}{|l|}{ Primary Treatment $\mathrm{e}^{\mathrm{s}}$} \\
\hline Substance abuse only & $1057(89.7)$ & $362(90.0)$ & $505(89.5)$ & $190(89.2)$ \\
\hline Substance abuse and mental health & $114(9.7)$ & $37(9.2)$ & $54(9.6)$ & $23(10.8)$ \\
\hline \multicolumn{5}{|l|}{ Family History of Substance Use ${ }^{f}$} \\
\hline Parents abused substances ${ }^{e}$ & $519(44.0)$ & $181(45.0)$ & $255(45.2)$ & $83(39.0)$ \\
\hline Other relative abused substances ${ }^{e}$ & $440(37.2)$ & $157(39.1)$ & $207(36.7)$ & $76(35.7)$ \\
\hline
\end{tabular}

${ }^{a} p<0.05 ; \quad{ }^{b} p<0.01 ; \quad{ }^{c} p<0.001 ;{ }^{d} p<0.10 ;$ eNot significant; ${ }^{f}$ Multiple responses Missing Values: ${ }^{1}=128,{ }^{2}=19,{ }^{3}=22,{ }^{4}=1,{ }^{5}=8$

use, roughly $64 \%$ and $52 \%$ reported their initiation between 13 and 18 years old, respectively. At intake, $56 \%$ of participants used multiple substances, rather than alcohol only (22\%) or a single illicit drug (22\%). The most commonly reported substance used at intake was alcohol, followed by cocaine, marijuana, opiates/narcotics, sedatives/ hypnotics, stimulants/ amphetamines, and then, hallucinogens/other drugs. Most participants were only being treated for substance use (90\%), and rest for a co-occurring substance use and mental health condition. Participants reported a history of substance abuse among their parents (44\%), siblings (32\%), and/or some other relative (37\%).

Most of the socio-demographic and substance abuse-related trends remained consistent across patterns of cigarette smoking initiation, except for their reported residence and drug use at intake. The majority of typical and atypical progressors lived in rural areas, whereas most never-smokers resided in urban areas (see Table 1).

\section{Atypical progressors vs. typical progressors}

Several findings were revealed in the adjusted model (see Table 2). As opposed to unadjusted model, in adjusted model gender, age of onset (i.e., 13 to 17 years age range vs. greater than 18 years), alcohol, opiates, and "other" drugs were no longer significant between atypical and typical progressors. Age of onset (i.e., less than 12 years old vs. greater than 18 years), number of substances used at admission (i.e., single illicit substance vs. alcohol only), and marijuana used at admission remained significant, while ethnicity, education, and the number of substances used at admission (i.e., multiple illicit substances vs. alcohol only) became significant in the adjusted model. In other words, ethnicity and education were the only significant demographic variables, and age of onset of substance use, the number/ type of drug use reported at admission, and marijuana use reported at admission were the significant substance-related variables. African Americans were more likely to be atypical progressors $(\mathrm{OR}=1.85$; 95\% $\mathrm{CI}=1.19-2.89, \mathrm{p}<0.01)$. Participants with a high school degree or less were more likely to be typical progressors $(\mathrm{OR}=0.61 ; 95 \% \mathrm{CI}=0.4$ $0.91, \mathrm{p}<0.05)$, compared to college-educated participants. Similar to the unadjusted model, participants who began using substances before 12 years of age were 8.77 times more likely to be an atypical progressor. Concerning the number of substances used at admission, participants who reported using either one illicit substance or multiple substances, rather than alcohol only, were more likely to be typical progressors, $(\mathrm{OR}=0.24 ; 95 \% \mathrm{CI}=0.12-0.46, \mathrm{p}<0.001)$ and $(\mathrm{OR}=0.50 ; 95 \% \mathrm{CI}=0.27-0.93, \mathrm{p}<0.05)$, respectively. Regarding the type of substance use at admission, marijuana users were more likely to be atypical progressors $(\mathrm{OR}=2.36$; $95 \% \mathrm{CI}=1.55-3.58, \mathrm{p}<0.001)$. 
Citation: Pirkey P, Kedia S, Webb L, Relyea G, Alam MM, et al. Cigarette Smoking Initiation among Substance Abuse Treatment Population: How applicable is the Gateway Hypothesis? J Addiction Prevention. 2015;3(2): 7.

ISSN: $2330-2178$

Table 2: Multivariate analysis.

\begin{tabular}{|c|c|c|c|c|}
\hline & \multicolumn{2}{|c|}{ Atypical vs. Typical Progressors } & \multicolumn{2}{|c|}{ Never-smokers vs. Typical Progressors } \\
\hline & $\begin{array}{l}\text { Unadjusted } \\
\text { OR }(\mathrm{Cl})\end{array}$ & $\begin{array}{l}\text { Adjusted } \\
\text { OR (CI) }\end{array}$ & $\begin{array}{l}\text { Unadjusted } \\
\text { OR }(\mathrm{Cl})\end{array}$ & $\begin{array}{l}\text { Adjusted } \\
\text { OR (CI) }\end{array}$ \\
\hline \multicolumn{5}{|l|}{ Demographics } \\
\hline \multicolumn{5}{|l|}{ Gender } \\
\hline Male vs. Female (ref) & $1.38(1.08,1.80)^{\mathrm{a}}$ & $1.06(0.77,1.47)$ & $1.72(1.19,2.49)^{b}$ & $1.34(0.89,2.02)$ \\
\hline \multicolumn{5}{|l|}{ Ethnicity } \\
\hline $\begin{array}{l}\text { African American } \\
\text { vs. Caucasian (ref) }\end{array}$ & $1.29(0.94,1.77)$ & $1.85(1.19,2.89)^{b}$ & $2.19(1.50,3.20)^{c}$ & $2.18(1.32,3.60)^{b}$ \\
\hline \multicolumn{5}{|l|}{ Education } \\
\hline $\begin{array}{l}\text { Middle/High School vs. } \\
\text { College (ref) }\end{array}$ & $0.80(0.57,1.12)$ & $0.61(0.41,0.91)^{\mathrm{a}}$ & $0.54(0.36,0.82)^{b}$ & $0.45(0.28,0.71)^{c}$ \\
\hline Unknown vs. College (ref) & $0.74(0.45,1.23)$ & $0.62(0.35,1.11)$ & $0.89(0.50,1.60)$ & $0.77(0.41,1.46)$ \\
\hline Urban vs. Rural (ref) & $1.01(0.78,1.31)$ & $0.97(0.69,1.35)$ & $0.58(0.42,0.81)^{b}$ & $1.38(0.92,2.09)$ \\
\hline \multicolumn{5}{|l|}{ Substance abuse-related Factors: } \\
\hline \multicolumn{5}{|l|}{ Age of onset of substance use } \\
\hline $6-12$ vs. $>18$ yrs (ref) & $12.70(7.94,20.32)^{c}$ & $8.77(0.51,14.98)^{\mathrm{c}}$ & $2.57(1.43,4.62)^{b}$ & $2.27(1.13,4.52)^{\mathrm{a}}$ \\
\hline $13-17$ vs. $>18$ yrs (ref) & $5.21(3.79,7.17)^{c}$ & $3.41(2.34,4.98)$ & $1.96(1.37,2.81)^{\mathrm{b}}$ & $1.77(1.15,2.72)^{\mathrm{b}}$ \\
\hline \multicolumn{5}{|l|}{ Number of substances used at admission } \\
\hline $\begin{array}{l}\text { Single illicit drug only vs. } \\
\text { Alcohol only (ref) }\end{array}$ & $0.21(0.14,0.32)^{\mathrm{c}}$ & $0.24(0.12,0.46)^{c}$ & $0.52(0.33,0.82)^{b}$ & $0.80(0.35,1.81)$ \\
\hline $\begin{array}{l}\text { Multiple substances vs. } \\
\text { Alcohol only (ref) }\end{array}$ & $0.94(0.67,1.31)$ & $0.50(0.27,0.93)^{\mathrm{a}}$ & $0.59(0.39,0.91)^{\mathrm{a}}$ & $0.78(0.34,1.80)$ \\
\hline \multicolumn{5}{|l|}{ Drug use reported at admission (No ref) } \\
\hline Alcohol & $0.31(2.38,4.09)^{c}$ & $1.36(0.84,2.20)$ & $1.72(1.23,2.40)^{b}$ & $1.12(0.57,2.23)$ \\
\hline Marijuana & $2.76(2.08,2.65)^{c}$ & $2.36(1.55,3.58)^{c}$ & $1.62(1.13,2.32)^{\mathrm{b}}$ & $1.51(0.86,2.65)$ \\
\hline Opiates/ narcotics & $0.59(0.44,0.81)^{c}$ & $1.05(0.66,1.66)$ & $0.48(0.31,0.74)^{\mathrm{b}}$ & $0.72(0.37,1.40)$ \\
\hline Sedatives/hypnotics & $0.76(0.51,1.12)$ & $0.74(0.45,1.23)$ & $0.61(0.35,1.07)$ & $0.86(0.42,1.78)$ \\
\hline Stimulants/ amphetamines & $0.68(0.46,1.02)$ & $0.88(0.51,1.53)$ & $0.35(0.18,0.69)^{b}$ & $0.49(0.22,1.08)$ \\
\hline Hallucinogens & $2.15(0.43,10.69)$ & $1.36(0.23,8.09)$ & $0.64(0.27,13.55)$ & $2.21(0.36,18.64)$ \\
\hline Other & $2.84(1.22,6.58)^{\mathrm{a}}$ & $3.43(1.29,9.15)$ & $3.07(1.17,8.04)^{\mathrm{a}}$ & $2.77(0.90,8.46)^{d}$ \\
\hline \multicolumn{5}{|l|}{ Primary treatment } \\
\hline $\begin{array}{l}\text { Substance abuse only vs. } \\
\text { Substance abuse \& Mental Health (ref) }\end{array}$ & $0.96(0.62,1.48)$ & $0.92(0.55,1.55)$ & $0.84(0.49,1.46)$ & $0.79(0.43,1.45)$ \\
\hline \multicolumn{5}{|l|}{ Family History of Substance Use } \\
\hline Parents abused substances & $1.01(0.78,1.30)$ & $0.77(0.56,1.06)$ & $0.78(0.56,1.09)$ & $0.71(0.48,1.06)^{d}$ \\
\hline Sibling abused substances & $0.96(0.73,1.26)$ & $0.94(0.68,1.31)$ & $0.88(0.61,1.26)$ & $1.10(0.73 .1 .65)$ \\
\hline Other relative abused substances & $0.91(0.70,1.18)$ & $0.85(0.62,1.16)$ & $0.87(0.61,1.22)$ & $0.84(0.57,1.24)$ \\
\hline
\end{tabular}

${ }^{a} p<0.05 ;{ }^{b} p<0.01 ;{ }^{c} p<0.001 ;{ }^{d} p<0.10$

\section{Never-smokers vs. typical progressors}

Similarly, several variables were significant in the adjusted model when comparing never smoker with typical progressors (see Table 2). Gender, residence, number of substances used at admission, and reported drug used at admission (i.e., alcohol, marijuana, opiates/ narcotics, and "other drugs") were not significant in the adjusted model. Ethnicity, education, age of onset of substance use, and cocaine remained significant. As shown in Table 2, African Americans were twice $(\mathrm{OR}=2.18 ; 95 \% \mathrm{CI}=1.32-3.60, \mathrm{p}<0.01)$ as likely to report being a never-smoker, compared to whites. College-educated participants were more likely to be never-smokers $(\mathrm{OR}=0.45 ; 95 \% \mathrm{CI}=0.28-0.71$, $\mathrm{p}<0.001)$. Individuals who began using substances, either before 12 years of age or between 13 to 17 years of age, were 2.27 and 1.77 times more likely to report being a never-smoker $(\mathrm{OR}=2.27 ; 95 \%$ $\mathrm{CI}=1.13-4.52, \mathrm{p}<0.05$ and $(\mathrm{OR}=1.77 ; 95 \% \mathrm{CI}=1.15-2.72, \mathrm{p}<0.01$, correspondingly) compared to those who began after 18 years of age. Participants who reported cocaine use at admission were more likely 
to report typical progression $(\mathrm{OR}=0.52 ; 95 \% \mathrm{CI}=0.29-0.94, \mathrm{p}<0.05)$.

\section{Discussion}

This study examined cigarette smoking initiation patterns among a substance abuse treatment population. Two important findings emerged: First, the high prevalence of cigarette smoking among this substance abuse treatment population (82\%), regardless of whether or not it was a gateway substance, supports the critical need to provide smoking cessation intervention among these individuals. Second, a sizable proportion of participants reported deviations from the GH (66\%: $48 \%$ atypical progressors and 18\% never-smokers), which is a larger percentage than reported from the general population $[12,29,30]$. Since the GH is only able to accommodate cigarette smoking progression in about one-third of this subpopulation, particularly among non-Hispanic whites and participants with less than a high school education, the GH framework is seemingly insufficient for explaining the progression of substance abuse among the majority of those with serious substance abuse problems when considering cigarettes as the gateway substance. Other studies have also noted the non-existent effect of alcohol as a gateway substance among substance use treatment clients [31].

The current study explored some of the factors -specifically, sociodemographics and substance abuse-related factors- that characterize those who diverge from the sequential, gateway pattern of progression (i.e., typical progression). The final models revealed demographic and substance abuse-related similarities when comparing typical progressors to both atypical progressors and never-smokers. Both adjusted models found that substance users who reported either being atypical progressors or never-smokers were more likely to have been African American and college-educated. The ethnicity-atypical/ never-smoker association parallels other studies involving substance use participants $[25,32,33]$. Ethnicity seems to be a well-studied variable, though education level does not, which is surprising given it has been purported to be the strongest demographic determinant for increased cigarette smoking [34]. The initiation of cigarette smoking, on the other hand, may differ with respect to the social surroundings and/or risk perception of college-educated substance abusers. For instance, being an atypical progressor may reflect the wide availability of other substances on college campuses for those who begin experimenting with drugs in college. A systematic review revealed that prior illicit drug use increases the likelihood of cigarette smoking initiation among college students, especially when their social groups accept cigarette smoking as a normative or safe behavior [35]. However, the college-educated participants who were identified as never-smokers may be more inclined to view tobacco as a taboo or risky/unhealthy practice and, in turn, use a more socially acceptable drug such as alcohol or even marijuana.

Several substance abuse-related factors were significant and varied based on the absence or presence of cigarette smoking. When comparing the younger age ranges for substance use onset (i.e., 6 to 12 years and 13 to 17 years old) to the older age range for substance use onset (i.e., 18 or older), individuals who initiated licit or illicit drug use at an earlier age were more likely to be either atypical progressors or never-smokers than typical progressors. Given the high prevalence of an early age of onset of substance use among all participants and the increased likelihood among atypical progressors and never-smokers, prevention efforts should focus on general substance use among individuals well before age 17, especially in high school. Also, assessing the initial preference for or the availability of a particular drug used and reported at admission may aid in explaining the varying progression patterns. In fact, marijuana was statistically significant for predicting atypical progressors. These findings concur with mounting evidence, which supports the notion that marijuana may be becoming customary for substance use onset and in turn, a problematic substance behavior. Unlike marijuana, reported cocaine use at admission was less likely to deviate in drug progression.

This study is not without limitations. First, the study participants were not randomly selected, so the study findings cannot be generalized. Second, the accuracy of retrospective reports may be questionable. However, previous studies have shown that age of onset has decent reliability. Recollection of age tends to shift upward as participant age increases. Regardless, the data were derived from participants' self-reports, which could be subject to this bias. Third, only participants who consented to participate in the study at substance abuse treatment intake and who could also be reached six months after admission were interviewed, which excluded persons who could not be reached by phone. Also, substance abuse participants who are treated in state-funded facilities are a specific subset of substance abusers seeking treatment. While two thirds of problematic substance use participants enroll in publicly-funded treatment facilities, future research should still explore a variety of treatment facilities [25]. Furthermore, problematic substance users currently not in treatment should be included in the future studies; however, this subset of abusers is relatively difficult to access.

In spite of these limitations, the implications of the findings are compelling. First, the prevalence of smoking, regardless of whether or not it was a "gateway" among substance abuse participants, supports the need to include role of smoking in future studies in this population. Second, the increased prevalence of atypical progressors and never-smokers suggests that the GH is an insufficient theory, especially among problematic substance users. This study highlights the necessary addition and exploration of other social and cognitive/ affective variables known to influence problematic substance use behavior, to aid in the development of targeted substance use prevention programs [2,36]. Exploring the variables associated with problematic behavior may begin to provide a more comprehensive understanding of the etiologic factors on substance use, progression, and predisposition. Likewise, research should evaluate polydrug vs. single use of each of the "softer" drug (i.e., tobacco, alcohol, and marijuana), and other illicit drugs (e.g., cocaine and opiates), among both problematic and experimental substance users to identify potential drug-specific etiologic variability. Other factors such as problematic behavior and social determinants should be assessed among substance abusers to elucidate the etiologic pathways for patterns of cigarette smoking initiation. The findings from this comparison may enable the development of tailored preventative programs for those at greatest risk with strategies to ward off problematic use of gateway substances preceding substance abuse and/or dependence.

\section{References}

1. Kandel DB (1992) Epidemiological trends and implications for understanding 
Citation: Pirkey P, Kedia S, Webb L, Relyea G, Alam MM, et al. Cigarette Smoking Initiation among Substance Abuse Treatment Population: How applicable is the Gateway Hypothesis? J Addiction Prevention. 2015;3(2): 7.

the nature of addiction. Res Publ Assoc Res Nerv Ment Dis 70: 23-40.

2. Vanyukov MM, Tarter RE, Kirillova GP, Kirisci L, Reynolds MD, et al. (2012) Common liability to addiction and "gateway hypothesis": theoretical, empirical and evolutionary perspective. Drug Alcohol Depend 123 Suppl 1: S3-S17.

3. Kandel D (1975) Stages in adolescent involvement in drug use. Science 190: 912-914.

4. Agrawal A, Grant JD, Waldron M, Duncan AE, Scherrer JF, et al. (2006) Risk for initiation of substance use as a function of age of onset of cigarette, alcohol and cannabis use: findings in a Midwestern female twin cohort. Prev Med 43: 125-128.

5. Bentler PM, Newcomb MD, Zimmerman MA (2002) Cigarette use and drug use progression: Growth trajectory and lagged effect hypotheses. In: Kande DB (Eds), Stages and pathways of drug involvement. Examining the gateway hypothesis. Cambridge University Press, Cambridge, pp. 223-253.

6. Kandel DB, Yamaguchi K, Chen K (1992) Stages of progression in drug involvement from adolescence to adulthood: further evidence for the gateway theory. J Stud Alcohol 53: 447-457.

7. Lewinsohn PM, Rohde P, Brown RA (1999) Level of current and past adolescent cigarette smoking as predictors of future substance use disorders in young adulthood. Addiction 94: 913-921.

8. Compton WM, Dawson DA, Conway KP, Brodsky M, Grant BF (2014) Transitions in illicit drug use status over 3 years: a prospective analysis of a general population sample. Am J Psychiatry 170: 660-670.

9. Degenhardt L, Dierker L, Chiu WT, Medina-Mora ME, Neumark Y, et al. (2010) Evaluating the drug use "gateway" theory using cross-national data: consistency and associations of the order of initiation of drug use among participants in the WHO World Mental Health Surveys. Drug Alcohol Depend 108: 84-97.

10. Donovan JE, Jessor R, Costa FM (1991) Adolescent health behavior and conventionality-unconventionality: an extension of problem-behavior therapy. Health Psychol 10: 52-61.

11. Mackesy-Amiti ME, Fendrich M, Goldstein PJ (1997) Sequence of drug use among serious drug users: typical vs atypical progression. Drug Alcoho Depend 45: 185-196.

12. Richter KP, Ahluwalia HK, Mosier MC, Nazir N, Ahluwalia JS (2002) A population based study of cigarette smoking among illicit drug users in the United States. Addiction 97: 861-869.

13. Whitesell NR, Kaufman CE, Keane EM, Crow CB, Shangreau C, et al. (2012) Patterns of substance use initiation among young adolescents in a Northern Plains American Indian tribe. Am J Drug and Alcohol Abuse 38: 383-388.

14. Kandel DB (2003) Does marijuana use cause the use of other drugs? JAMA 289: 482-483.

15. Furr-Holden CD, lalongo NS, Anthony JC, Petras H, Kellam SG (2004) Developmentally inspired drug prevention: middle school outcomes in a school-based randomized prevention trial. Drug Alcohol Depend 73: 149-158.

16. Guydish J, Passalacqua E, Pagano A, Martínez C, Le T, et al. (2015) An international systematic review of smoking prevalence in addiction treatment. Addiction [Epub ahead of print].

17. Guydish J, Passalacqua E, Tajima B, Chan M, Chun J, et al. (2011) Smoking prevalence in addiction treatment: a review. Nicotine Tob Res 13: 401-411.

18. Lasser K, Boyd JW, Woolhandler S, Himmelstein DU, McCormick D, et al. (2000) Smoking and mental illness: A population-based prevalence study. JAMA 284: 2606-2610.

19. Kalman D, Kim S, DiGirolamo G, Smelson D, Ziedonis D (2010) Addressing tobacco use disorder in smokers in early remission from alcohol dependence: the case for integrating smoking cessation services in substance use disorder treatment programs. Clin Psychol Rev 30: 12-24.
20. Bobo JK, Gilchrest LD, Schilling RF 2nd, Noach B, Schinke SP (1987) Cigarette smoking cessation attempts by recovering alcoholics. Addict Behav 12: $209-215$.

21. Joseph AM, Willenbring ML, Nugent SM, Nelson DB (2004) A randomized trial of concurrent versus delayed smoking intervention for patients in alcohol dependence treatment. J Stud Alcohol 65: 681-691.

22. Rohsenow DJ, Colby SM, Martin RA, Monti PM (2005) Nicotine and other substance interaction expectancies questionnaire: relationship of expectancies to substance use. Addict Behav 30: 629-641.

23. Hser YI, McCarthy WJ, Anglin MD (1994) Tobacco use as a distal predictor of mortality among long-term narcotics addicts. Prev Med 23: 61-69.

24. Hurt RD, Offord KP, Croghan IT, Gomez-Dahl L, Kottke TE, et al. (1996) Mortality following inpatient addictions treatment: Role of tobacco use in a community-based cohort. JAMA 275: 1097-1103.

25. U.S. Department of Health and Human Services (USHHS) (2011) Results from the 2010 National Survey on Drug Use and Health: Summary of national findings (NSDUH Series H-41, HHS Publication No. (SMA) 11-4658) Rockville, MD: Substance Abuse and Mental Health Services Administration.

26. McLellan AT, Kushner H, Metzger D, Peters R, Smith I, et al. (1992) The fifth edition of the Addiction Severity Index. J Subst Abuse Treat 9: 199-213.

27. McLellan AT, Cacciola JC, Alterman AI, Rikoon SH, Carise D (2006) The Addiction Severity Index at 25: origins, contributions and transitions. Am J Addict 15: 113-124.

28. Ward KD, Kedia S, Webb L, Relyea GE (2012) Nicotine dependence among clients receiving publicly funded substance abuse treatment. Drug Alcohol Depend 125: 95-102.

29. Golub A, Johnson BD (2002) The misuse of the 'gateway theory' in US policy on drug abuse control: a secondary analysis of the muddled deduction. Int $J$ Drug Policy 13: 5-19.

30. Lai S, Lai H, Page JB, McCoy CB (2000) The association between cigarette smoking and drug abuse in the United States. J Addict Dis 19: 11-24.

31. Golub A, Johnson BD (1994) The shifting importance of alcohol and marijuana as gateway substances among serious drug abusers. J Stud Alcohol 55: 607614

32. Benowitz NL, Bernert JT, Caraballo RS, Holiday DB, Wang J (2009) Optima serum cotinine levels for distinguishing cigarette smokers and nonsmokers within different racial/ethnic groups in the United States between 1999 and 2004. Am J Epidemiol 169: 236-248.

33. Coleman-Cowger VH, Catlin ML (2013) Changes in tobacco use patterns among adolescents in substance abuse treatment. J Subst Abuse Treat 45: 227-234.

34. Green MP, McCausland KL, Xiao H, Duke JC, Vallone DM, et al. (2007) A closer look at smoking among young adults: where tobacco control should focus its attention. Am J Public Health 97: 1427-1433.

35. Freedman KS, Nelson NM, Feldman LL (2012) Smoking initiation among young adults in the United States and Canada, 1998-2010: a systematic review. Prev Chronic Dis 9: E05.

36. Degenhardt L, Chiu WT, Conway K, Dierker L, Glantz M, et al. (2009) Does the 'gateway' matter? Associations between the order of drug use initiation and the development of drug dependence in the National Comorbidity Study Replication. Psychol Med 39: 157-167.

\section{Acknowledgements}

This research was funded by the USA federal agency, Center for Substance Abuse Treatment - Substance Abuse and Mental Health Services (CSATSAMHSA). Part of the financial assistance also came from The University of Memphis Faculty Research Grant. The authors would like to thank the Division of Alcohol and Drug Abuse Services of the Tennessee Department of Mental Health for their support of this research. The authors appreciate the treatment facilities and clients who participated in this study. 